\title{
The Influence of Growth Substrate and Capacity for Oxidative Phosphorylation on Respiratory Oscillations in Synchronous Cultures of Escherichia coli $\mathrm{K} 12$
}

\author{
By R. K. POOLE \\ Department of Microbiology, Queen Elizabeth College (University of London), \\ Campden Hill, London W8 7 AH
}

(Received 27 October 1976)

\begin{abstract}
SUMMARY
Fluctuations in cell volume during exponential growth of Escherichia coli KI2 changed the effectiveness of the continuous-flow centrifugation method for preparing synchronous cultures. Rates of oxygen uptake in synchronous cultures were measured using an electrode system open to the atmosphere. In synchronous cultures of both the parental strain and an adenosine triphosphatase-deficient mutant, which was incapable of oxidative phosphorylation, respiration rates doubled during the cell cycle but oscillated with a periodicity of approximately half a cycle. Synchronous cultures of the parental strain growing on glycerol and Casamino acids showed a stepwise pattern of oxygen consumption. Continuous flow centrifugation did not markedly affect the increases in the numbers and respiration rates of cells in synchronous cultures. Respiratory oscillations also occurred on inoculation of a late-stationary phase culture into fresh medium, although synchronous division was not observed. The possible mechanisms underlying respiratory fluctuations under different growth conditions are discussed.
\end{abstract}

\section{INTRODUCTION}

Rates of respiration during the cell cycles of a variety of eukaryotic micro-organisms have been extensively studied (see Lloyd, 1974) but the biochemical and physiological bases for the complex patterns that are often observed are poorly understood. During synchronous growth in a defined medium containing alanine as carbon source after selection by continuous-flow centrifugation, respiration rates of Escherichia coli oscillated with a periodicity of about one-third of a cell cycle (Evans, 1975). In this paper the physiological basis of these oscillations has been studied by examining the effects of (i) growth with alternative carbon sources and (ii) a genetic lesion in the membrane-bound adenosine triphosphatase (ATPase). It is also shown that respiratory oscillations may persist independently of synchronous cell growth and division. Parts of this work have been briefly communicated previously (Poole, 1975).

\section{METHODS}

Organisms and growth conditions. Escherichia coli strains AI002 and AI03c were kindly supplied by Dr B. A. Haddock. Strain AI002 ( $\mathrm{KI} 2$ Y mel ilv lacI- met $E^{-}$) is the parental strain from which the ATPase-deficient mutant, A I03c, was derived (Schairer \& Haddock, I972). Both were grown at $37^{\circ} \mathrm{C}$ in mineral salts medium (Poole, 1977) containing either $0.5 \%(\mathrm{w} / \mathrm{v})$ glucose or $0.5 \%(\mathrm{w} / \mathrm{v})$ glycerol as carbon source, in the absence or presence of $0.1 \%(w / v)$ vitamin-free Casamino acids (Difco). Strain AI03c was regularly screened for 
reversion by checking its inability to grow on solid defined medium containing only acetate, malate and succinate (all at $0.2 \%, w / v$ ) as carbon sources (Schairer \& Haddock, 1972). Glucose-containing cultures (2 l) were grown under forced aeration and with magnetic stirring. Glycerol-containing cultures were grown with more vigorous aeration by shaking $200 \mathrm{ml}$ volumes in I 1 conical flasks at $200 \mathrm{rev}$. $\mathrm{min}^{-1}$. Synchronous cultures $(200 \mathrm{ml})$ were grown, with magnetic stirring, in an open vessel fitted with a heating coil. Alternatively, $2 \mathrm{ml}$ portions of concentrated culture were grown in the open reaction vessel of an oxygen electrode, as described below.

Preparation of synchronous cultures by continuous-flow centrifugation. This was as described previously (Poole, 1977). The total centrifugal force $(g$-min) was calculated for a radius of $2.9 \mathrm{~cm}$ (half-maximal radius) using the mean residence time of the culture in the rotor (rotor volume, $\mathrm{ml} /$ flow rate, $\mathrm{ml} \mathrm{min}^{-1}$ ). The resulting synchronous culture was used either directly or after concentration by rapid filtration under reduced pressure through a $50 \mathrm{~mm}$ diam. Nuflow membrane filter $(0.45 \mu \mathrm{m}$ pore size; Oxoid). Cells were recovered quantitatively on the filter and immediately resuspended in a small volume of the filtrate. Zero time for the synchronous culture was taken as the time when half the effluent volume subsequently used for analysis had been collected.

Cell numbers and volumes. These were determined using a Coulter counter model $\mathrm{Z}_{\mathrm{B1}}$ and Channelyzer Crooo (Coulter Electronics, Harpenden, Hertfordshire) as described by Poole (1977).

Assessment of synchrony. Synchrony indices calculated for the first $\left(F_{1}\right)$ and second $\left(F_{2}\right)$ increases in cell numbers (midpoints indicated by vertical lines in the figures) are those of Blumenthal \& Zahler (I962). For an exponentially growing culture, the value of the index is 0 ; for a theoretical culture exhibiting perfect synchrony, its value is $\mathrm{I} \cdot 0$.

Measurements of oxygen uptake. Oxygen uptake rates in $2 \mathrm{ml}$ culture samples were measured using an $\mathrm{O}_{2}$ electrode (Rank Bros, Bottisham, Cambridge) calibrated as described by Chappell (1964). Respiratory inhibitors and uncoupling agents were added as small volumes of concentrated aqueous or methanolic solutions; control experiments showed that these concentrations of methanol alone had no effect on respiration rates. Possible errors in the determination of respiratory patterns in rapidly growing cultures, arising from infrequent sampling, were overcome by obtaining a continuous recording of $\mathrm{O}_{2}$ tension in small cultures in an $\mathrm{O}_{2}$ electrode system open to the atmosphere, similar to that described by Degn, Lilleør \& Iversen (1973). The standard reaction vessel (Rank Bros) was a cylindrical well, $1.6 \mathrm{~cm}$ diam. $\times 4.0 \mathrm{~cm}$ depth, at the bottom of which was a Clark-type $\mathrm{O}_{2}$ electrode and a magnetic stirrer bar. The cap that normally seals the contents of the vessel from the atmosphere was replaced with a bung fitted with inlet and outlet tubes through which a slow stream of water-saturated air was passed to minimize evaporation from the sample. The maximum rate of evaporation for a vigorously stirred $2 \mathrm{ml}$ culture sample was about $3 \times 10^{-3} \mathrm{ml} \mathrm{min}^{-1}$ at $35^{\circ} \mathrm{C}$. The $\mathrm{O}_{2}$ transfer constant, $K$, was determined from the observed half-time of the first-order equilibration of liquid (made anoxic with a grain of dithionite) with atmospheric $\mathrm{O}_{2}$ (Degn et al., 1973). $K$ was measured in each experiment; the values were in the range 0.41 to $0.51 \mathrm{~min}^{-1}$.

When temperature, sample volume and rate of stirring are constant, the measured variations in dissolved $\mathrm{O}_{2}$ tension reflect the changing respiration rates of cells in the culture. In a control experiment, $2 \mathrm{ml}$ of air-saturated growth medium without cells was incubated for 150 min under the conditions described above; fluctuations in the recorded $\mathrm{O}_{2}$ tension were less than $2 \%$ of the total over this period. Samples (Io $\mu \mathrm{l}$ ) of culture were removed from a second identical vessel and diluted in electrolyte for determinations of cell numbers. 


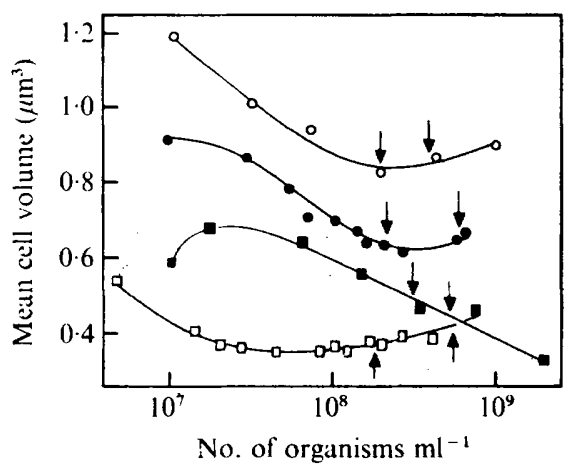

Fig. I

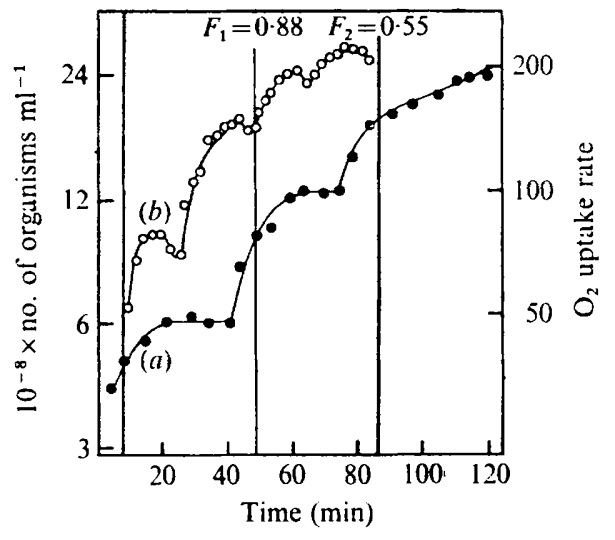

Fig. 2

Fig. I. Mean cell volume as a function of cell population during asynchronous growth of $E$. coli strains AIOO2 and AIO3C in media containing glucose or glycerol. Cell numbers and volumes were determined in formaldehyde-fixed (final concn $0.4 \%$ ) culture samples. $\bigcirc$, Strain AIO3c grown with glucose and Casamino acids, doubling time $48 \mathrm{~min} ; 0$, strain A1002 grown with glucose and Casamino acids, doubling time $44 \mathrm{~min}$; a, strain AI002 grown with glycerol and Casamino acids, doubling time $40 \mathrm{~min}$; $\square$ strain, AIOO2 grown with glycerol, no Casamino acids, doubling time $126 \mathrm{~min}$. Arrows indicate the limits of the periods during which the cultures were routinely used for continuous-flow centrifugation.

Fig. 2. Oxygen uptake in a synchronous culture of $E$. coli strain AI002 grown with glucose and Casamino acids. The population at the time of selection was $4.4 \times 10^{8}$ cells ml-1. Flow rate of the culture through the continuous action rotor was $161 \mathrm{ml} \mathrm{min}-1$ and the rotor speed $15900 \mathrm{rev} . \mathrm{min}^{-1}$ $\left(2.2 \times 10^{4} \mathrm{~g}\right.$-min at half-maximal radius). After discarding the first $300 \mathrm{ml}$ of effluent, a portion $(75 \mathrm{ml})$, which contained $6.9 \%$ of the original culture, was collected and concentrated $13-$ fold. Samples $(2 \mathrm{ml})$ were cultured in open $\mathrm{O}_{2}$ electrode vessels. - , Cell numbers; $\mathrm{O}, \mathrm{O}_{2}$ uptake rates calculated at intervals corresponding to $2.5 \mathrm{~min}$ from the polarographic trace and expressed as ng-atom $O \mathrm{~min}^{-1} \mathrm{ml}^{-1} . F_{1}$ and $F_{2}$ denote the synchrony indices of the first and second doublings of the cell numbers, respectively.

Table I. Effect of growth phase on the proportion of glucose-grown E. coli strain A1002 escaping retention in the continuous action rotor

$\begin{array}{cc}\begin{array}{c}\text { Cell population } \\ \text { when centrifuged } \\ \left(10^{-8} \times \text { organisms ml } l^{-1}\right)\end{array} & \begin{array}{c}10^{-4} \times \text { total centrifugal } \\ \text { force }(g-\text { min })\end{array} \\ 2 \cdot 1 & 2 \cdot 27 \\ 4 \cdot 4 & 2 \cdot 20 \\ 7 \cdot 3 & 2 \cdot 30\end{array}$

Extinction. The extinction of culture samples was measured in cuvettes of $\mathrm{I} \mathrm{cm}$ light path using a Pye Unicam SPI 700 spectrophotometer.

Chemicals. Carbonyl cyanide $m$-chlorophenyl hydrazone (CCCP) was from Sigma; dibromothymoquinone was a generous gift from Dr B. A. Haddock. All other reagents were of the highest purity commercially available.

\section{RESULTS}

Fluctuations in cell volume during asynchonous growth

During asynchronous growth of an unfractionated culture the mean cell volume fluctuated markedly (Fig. I). Cells of strains AIO02 and AI03c, grown with glucose and Casamino acids, decreased in volume until the population reached about $2 \times 10^{8}$ organisms $\mathrm{ml}^{-1}$ and then, fluctuating by less than $6 \%$ for three doublings, their volume increased slowly as the 


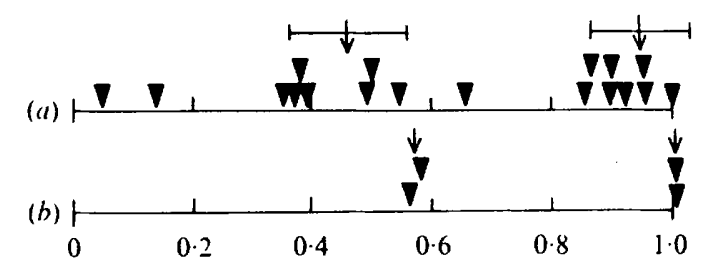

Fig. 3. Cell cycle maps of timings of peaks in $\mathrm{O}_{2}$ uptake during the cell cycle of $E$. coli grown with glucose and Casamino acids. In $(a)$ and $(b)$ the abscissae represent (as a linear scale) the cell cycle taken as the period between the midpoints of the first and second increases of cell numbers in synchronous cultures. The triangles $(\nabla)$ denote the timing of the peaks of $\mathrm{O}_{2}$ uptake during the cell cycle of $(a)$ strain AIOO2 and $(b)$ strain AI03c. Arrows with crossbars indicate mean values with standard deviations.

population approached $\mathrm{I} \times 10^{8}$ organisms $\mathrm{ml}^{-1}$. Cells grown with glycerol and Casamino acids continued to decrease in mean volume (about $0.07 \mu \mathrm{m}^{3}$ per doubling time) over the period examined; in the absence of Casamino acids, the fluctuation in cell volume was less marked. These results confirm and extend previous reports (Stephenson, 1949; Zaritsky \& Pritchard, 1973; Smither, 1975).

The effect of these fluctuations, and consequently of the rate of sedimentation, on the recovery of glucose-grown strain AIOO2 in the rotor effluent is shown in Table 1. Under otherwise similar conditions, the proportion of cells in the rotor effluent depended on the phase of growth, being inversely proportional to the cell population at the time of centrifuging. Strict standardization of the age and size of the inoculum and of the phase of growth at harvest (limited by the arrows in Fig. I) eliminated wide variations in the proportion of the original culture recovered.

\section{Oxygen uptake in synchronous cultures of strain A1002 grown with glucose and Casamino acids}

When the effluent culture from the continuous action rotor was concentrated 13 -fold and incubated in an open electrode vessel, the numbers of cells increased by $30 \%$ during the first $20 \mathrm{~min}$ of the experiment; thereafter two synchronous doublings of numbers occurred, with good synchrony indices (Fig. 2a). The first complete cell cycle (indicated by the vertical bars) took $42 \mathrm{~min}$. In seven similar experiments the mean value was $44 \mathrm{~min}$ (S.D. \pm 5.4 ), equal to the mean generation time of exponentially growing cultures.

The respiration rate, calculated from the continuous recorder trace of $\mathrm{O}_{2}$ concentration (Degn et al., 1973), doubled in a period equal to the length of a cycle, but rose to two maxima in each cell cycle, one just before the midpoint of cell division and the other in the first half of the cycle (Fig. $2 b$ ). Oscillations of the same periodicity were consistently observed in seven similar experiments. The mean positions of the peaks measured in ro cell cycles were 0.45 and 0.95 of the cycle (Fig. $3 a$ ). The amplitude of the oscillations (i.e. peak to trough) was more variable, ranging from 7 to $17 \%$ of the total respiration rate measured at the peaks.

Rates of $\mathrm{O}_{2}$ uptake were also measured (using a conventional oxygen electrode) in samples withdrawn at intervals from a larger synchronous culture that had not been concentrated by filtration (results not shown). Two experiments confirmed that the rates of respiration oscillated and doubled during the cell cycle. Using this method of analysis the respiratory discontinuities could not be fully resolved due to the relative infrequency with which samples could be removed and analysed. The amplitude of the oscillations was always greater at the beginning of the experiments and subsequently decreased as the degree of synchrony of growth decayed. 
A control experiment, described below, showed that growth and respiration of cultures were not affected by centrifugation, the short exposure to sub-optimal temperatures or rapid filtration. A culture $\left(2.7 \times 10^{8}\right.$ cells $\left.\mathrm{ml}^{-1}\right)$ was passed through the continuous action rotor, the centrifuge was stopped, and the thick cell suspension within the rotor was mixed with the effluent, which contained $5 \%$ of the initial population, and vigorously aerated at $37^{\circ} \mathrm{C}$. Exponential increases in the number of cells, the extinction of the culture and the $\mathrm{O}_{2}$ uptake rate (measured using a conventional $\mathrm{O}_{2}$ electrode system) were all similar to those before selection and showed no discontinuities. When such a culture was concentrated by filtration and incubated in the open electrode, a similar exponential increase in $\mathrm{O}_{2}$ uptake rate was observed.

\section{Oxygen uptake in a synchronous culture of the ATPase-deficient mutant}

Strain AI03c lacks the reversible membrane-bound $\mathbf{M g}^{2+}$-dependent ATPase and so cannot utilize a transmembrane proton motive force for ATP synthesis (Schairer \& Haddock, 1972). After continuous-flow selection, a lag of about $30 \mathrm{~min}$ preceded the onset of the first increase in the number of cells: the length of the subsequent complete cell cycle was $70 \mathrm{~min}$ (Fig. 4a). Though the rate of $\mathrm{O}_{2}$ uptake increased exponentially overall during the experiment, doubling during a cell cycle, it still oscillated (Fig. $4 b$ ). The first peak of uptake occurred before the onset of division in the population and the second coincided with the midpoint of division. This pattern was repeated in the next cycle; similar results were obtained in a second experiment. The observed patterns of respiration during the cell cycle of the mutant (Fig. $3 b$ ) are thus similar to those of its parental strain.

\section{Oxygen uptake in synchronous cultures of strain A1002 grown with glycerol and Casamino acids}

To investigate the contribution of catabolite repression by glucose to the observed oscillations, glycerol was used as an alternative carbon source. Respiration rates per cell in glycerol-containing cultures were two- to threefold higher than those of glucose-containing cultures. The length of the first complete cycle was identical to the mean generation time of exponential cultures (40 min). Synchronous growth was maintained for at least two complete cycles (Fig. $5 a$ ). In contrast to the oscillating rates of respiration observed in glucosecontaining cutures (Fig. $2 b$ ), stepped patterns of respiration were obtained (Fig. $5 b$ ). In measuring the periodicity of the steps, the inflections between the plateaus and the rapid rises (steps) in respiration rates were used as easily identifiable points. Using this convention the mean periodicity of the steps observed in two separate experiments (one of which is shown in Fig. 5) was 0.76 of a cell cycle (S.D. $\pm 0.1 \mathrm{I}$ ). This phenomenon has been observed previously by Scopes \& Williamson (1964) in synchronous cultures of Saccharomyces cerevisiae prepared by starvation. They suggested that the timing 'clock' for growth (reflected in respiration rates) may be dissociated from the control of cell division.

\section{Sensitivity of intact cells to respiratory inhibitors and uncoupling agents}

Titration of the varying respiration rates in synchronous cultures of eukaryotic microorganisms with inhibitors of electron transport or uncouplers of energy conservation has helped in understanding the mechanisms underlying the respiratory discontinuities (e.g. Poole, Lloyd \& Kemp, 1973). Similar experiments with $E$. coli were frustrated by the insensitivity or unreproducibility of the response of intact cells to a range of respiratory poisons. Although $\mathrm{KCN}$ (at $\mathrm{I} \cdot 5 \mathrm{mM}$ ) gave greater than $90 \%$ inhibition of $\mathrm{O}_{2}$ uptake by strain A1002 grown with glycerol, this was not achieved until 3 to $4 \mathrm{~min}$ after adding the 


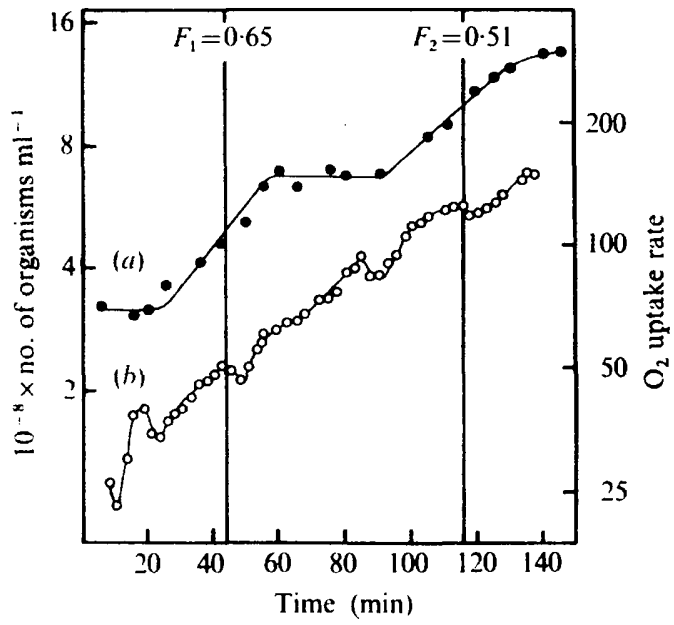

Fig. 4

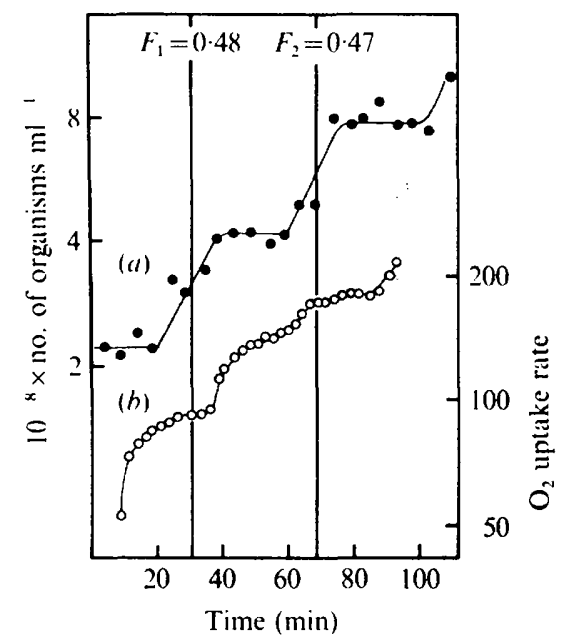

Fig. 5

Fig. 4. Oxygen uptake in a synchronous culture of $E$. coli strain Alo3c grown with glucose and Casamino acids. The population at the time of selection was $3.3 \times 10^{8}$ cells $\mathrm{ml}^{-1}$. Flow rate of the culture through the continuous action rotor was $164 \mathrm{ml} \mathrm{min}^{-1}$ and the rotor speed $14700 \mathrm{rev} . \mathrm{min}^{-1}$ $\left(\mathrm{I} .85 \times 10^{4} \mathrm{~g}\right.$-min at half-maximal radius). After discarding the first $300 \mathrm{ml}$ of effluent, a portion $(50 \mathrm{ml})$, which contained $10 \%$ of the original culture, was collected and concentrated I0-fold. Samples $(2 \mathrm{ml})$ were cultured in open $\mathrm{O}_{2}$ electrode vessels. Cell numbers; $O, \mathrm{O}_{2}$ uptake rates calculated at intervals corresponding to $2.5 \mathrm{~min}$ from the polarographic trace and expressed as $\mathrm{ng}$-atom $\mathrm{O} \mathrm{min}^{-1} \mathrm{ml}^{-1} . F_{1}$ and $F_{2}$ denote the synchrony indices of the first and second doublings of the cell numbers, respectively.

Fig. 5. Oxygen uptake in a synchronous culture of $E$. coli strain A1002 grown with glycerol and Casamino acids. The population at the time of selection was $3.3 \times 10^{8}$ cells ml-1. Flow rate of the culture through the continuous action rotor was $170 \mathrm{mlmin}^{-1}$ and the rotor speed $15000 \mathrm{rev} . \mathrm{min}^{-1}$ $\left(1.85 \times 10^{4} \mathrm{~g}\right.$-min at half-maximal radius). After discarding the first $300 \mathrm{ml}$ of effluent, a portion $(200 \mathrm{ml})$, which contained $8.6 \%$ of the initial population, was collected and concentrated 10 -fold. Samples $(2 \mathrm{ml})$ were cultured in open $\mathrm{O}_{2}$ electrode vessels., Cell numbers; $\mathrm{O}, \mathrm{O}_{2}$ uptake rates calculated at intervals corresponding to $2.4 \mathrm{~min}$ from the polarographic trace and expressed as ng-atom $O$ min $^{-1} \mathrm{ml}^{-1} . F_{1}$ and $F_{2}$ denote the synchrony indices of the first and second doublings of the cell numbers, respectively.

inhibitor. Dibromothymoquinone (at $0.5 \mathrm{~mm}$ ) had no effect on $\mathrm{O}_{2}$ uptake by intact cells up to $2 \mathrm{~min}$ after its addition; yet at $0.1 \mathrm{mM}$ it gives $20 \%$ inhibition of oxidase activity of membrane particles and at $0.5 \mathrm{~mm}$ totally inhibits growth (Poole \& Haddock, 1975). Occasionally, stimulation of respiration rates (20 to $50 \%$ ) was achieved by dibromophenol, 2,4-dinitrophenol or pentachlorophenol (all at Io $\mu \mathrm{M})$ or by CCCP $(2 \mu \mathrm{M})$. These effects were not reproducible and were of limited value in quantitative measurements of the sensitivity of cells at various stages of the cell cycle.

\section{Respiratory oscillations in the absence of synchronous growth}

Oscillations in the rate of $\mathrm{O}_{2}$ uptake were also observed after perturbation of growth conditions in the absence of synchronous growth. Cells from a $\mathrm{I} 6 \mathrm{~h}$ late-stationary phase culture were diluted in fresh medium containing glucose and Casamino acids. The number of cells increased gradually after 30 min incubation until the normal exponential growth rate was achieved after about 90 min (Fig. 6a). Although the increase in the extinction of the culture was discontinuous (Fig. $6 b$ ), there was no evidence of synchronous cell division. The induction of synchrony by transfer of stationary phase cells to fresh medium is critically 


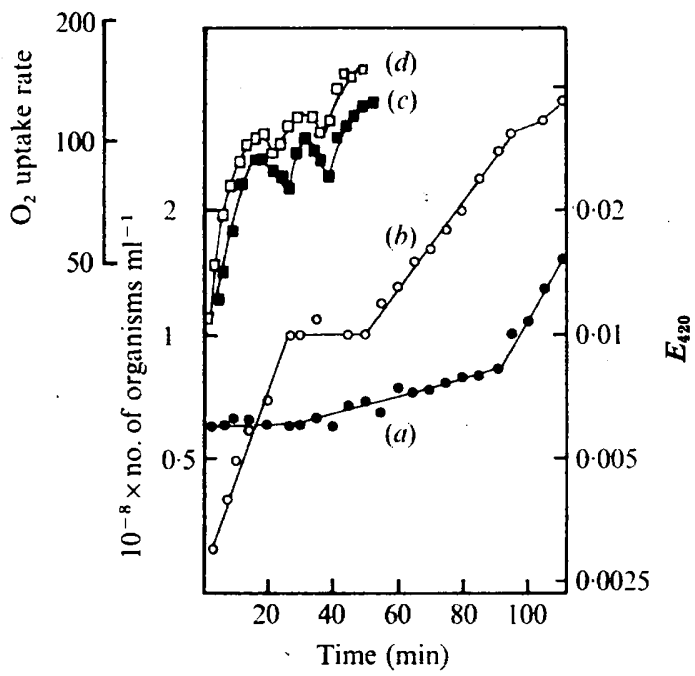

Fig. 6. Changes in cell population and respiration rate on inoculating a stationary phase culture of $E$. coli $\mathrm{Al} 002$ into fresh glucose-containing medium. Cell numbers $(O)$ and extinction $(O)$ were determined in formaldehyde-fixed samples removed from a culture which had been inoculated at a 1 : 400 dilution with cells from a stationary phase culture $\left(2 \times 10^{\circ}\right.$ cells $\left.\mathrm{ml}^{-1}\right)$. For determination of $\mathrm{O}_{2}$ uptake rates ( $\square, \square$; duplicate expts), the stationary phase culture was diluted 40 -fold and incubated in an open $\mathrm{O}_{2}$ electrode vessel: uptake rates are expressed as ng-atom $\mathrm{O} \mathrm{min}^{-1} \mathrm{ml}^{-1}$.

dependent on the period within the stationary phase when cells are taken (Cutler \& Evans, 1966). Oxygen uptake, measured in an open electrode system in a parallel experiment, oscillated markedly during the first $60 \mathrm{~min}$ of growth, with a periodicity of about $20 \mathrm{~min}$ (Fig. 6c). These oscillations were reproduced in a second experiment (Fig. 6d).

\section{DISCUSSION}

Despite its many advantages (Lloyd et al., 1975; Evans, 1975), continuous-flow age selection may result in extended cell cycle times (Fig. 4). Sometimes, the period between collection of the rotor effluent and the first synchronous division is considerably less than the length of subsequent cycles (Figs 4 and 5), or the first division is completed immediately after establishing the synchronous culture (Fig. 2). This is probably a result of the fluctuations in the buoyant density of cells that occur during the cell cycle of this organism (Poole, 1977). Furthermore, when the technique is applied to cultures grown with glycerol in the absence of Casamino acids, cells collected in the rotor effluent divided asynchronously with a doubling time considerably greater than that of the unfractionated culture (unpublished observations).

In constructing cell cycle maps (Fig. 3), the short periods preceding the first division have been regarded as unperturbed latter portions of the normal cycle, since control experiments showed that the continuous-flow procedure caused little disturbance of growth and respiration.

The measurement of respiration rates of cultures using a continuous recording of the $\mathrm{O}_{2}$ tension has obvious advantages where conventional methods of sampling and subsequent analysis offer insufficient time resolution. The continuous nature of these measurements makes repeated determinations on identical samples and the assessment of experimental 
precision difficult. However, the observed respiratory fluctuations were reproducible in replicate experiments and were much greater than the small fluctuations observed in the absence of respiring cells caused by changes in sample volume, temperature and stirring rate. The principal disadvantages of the method are: (i) the rate of stirring and thus of aeration must be chosen to maintain $\mathbf{O}_{2}$-sufficient growth conditions throughout the experiment, whilst allowing the changing respiration of cells to cause measurable changes in dissolved $\mathrm{O}_{2}$ tension; (ii) in order to avoid marked changes in culture volume, the number of cells must be determined in samples from a second vessel; and (iii) the effect of respiratory inhibitors on $\mathrm{O}_{2}$ uptake rates at various stages of growth cannot be studied without also inhibiting growth.

In this study two peaks of $\mathrm{O}_{2}$ uptake, rather than three (Evans, 1975), were observed during each cell cycle and these were replaced by a stepped pattern of uptake in cultures grown with glycerol, a non-catabolite-repressive substrate. These observations resemble those obtained with the highly-fermentative fission yeast Schizosaccharomyces pombe in three respects. (i) During the cell cycle of cells grown with glucose, the respiratory activity oscillates with a periodicity of half a cycle (Poole et al., I973). (ii) Growth in the presence of a non-catabolite-repressive carbon source, glycerol, results in stepwise increases in $\mathrm{O}_{2}$ uptake (Poole \& Lloyd, 1974). (iii) Oscillations observed with glucose as carbon source do not appear to be due to respiratory control in vivo, i.e. the rate limitation of $\mathrm{O}_{2}$ consumption by the availability of ADP in the presence of $P_{1}$ (Chance \& Williams, 1956). This conclusion is supported, in the case of $E$. coli, by the persistence of the oscillations in a mutant incapable of oxidative phosphorylation and, in $S$. pombe, by the finding that CCCP preferentially stimulates rates of $\mathrm{O}_{2}$ uptake and heat evolution when respiration rates are at a maximum rather than a minimum (Poole et al., 1973).

Two explanations seem possible for the persistence of respiratory oscillations after inoculation of stationary phase cells into fresh growth medium. First, the events responsible for their generation may be components of a sequence which is normally tightly coupled to the cycle of growth and division. These cycles correspond in principle to the 'growth cycle' and the 'DNA-division cycle', respectively, proposed by Mitchison (1971). Under appropriate conditions of metabolic perturbation (in this case transition from the stationary phase to the growing state) the two sequences or cycles may be dissociated. Alternatively, the oscillations may reflect the functioning of complex biochemical regulatory mechanisms which are triggered by the onset of the stationary phase, and which are not causally related to any sequence constituting the normal cell cycle. Similar results have recently been obtained with the yeast Candida utilis, where the onset of the stationary phase due to depletion of the carbon source triggers an oscillation in respiration rates (D. Lloyd \& R. K. Poole, unpublished results). Further characterization and comparison of the oscillations resulting from metabolic perturbation and those observed during the normal cell cycle will be necessary to distinguish between these two possibilities.

I am grateful to Dr C. F. Thurston for helpful advice and discussion during the course of this work and for his critical reading of the manuscript. 


\section{REFERENCES}

BLumenthal, L. K. \& ZAHLER, S. A. (1962). Index for measurement of synchronization of cell populations. Science I35, 724.

ChANCE, B. \& Williams, G. R. (1956). The respiratory chain and oxidative phosphorylation. Advances in Enzymology 17, 65-134.

ChAPPELL, J. B. (1964). The oxidation of citrate, isocitrate and cis-aconitate by isolated mitochondria. Biochemical Journal 90, 225-237.

CUTLER, R. G. \& EVANs, J. E. (1966). Synchronization of bacteria by a stationary-phase method. Journal of Bacteriology 91, 469-476.

Degn, H., Lilleør, M. \& IVERsen, J. J. L. (1973). The occurrence of a stepwise-decreasing respiration rate during oxidative assimilation of different substrates by resting Klebsiella aerogenes in a system open to oxygen. Biochemical Journal 136, I097-I I04.

Evans, J. B. (1975). Preparation of synchronous cultures of Escherichia coli by continuous-flow size selection. Journal of General Microbiology 91, 188-190.

LLOYD, D. (1974). Environmental modification of mitochondrial composition and activities. In The Mitochondria of Micro-organisms, pp. 159-250. London: Academic Press.

Lloyd, D., John, L., Edwards, C. \& ChAGLA, A. H. (I975). Synchronous cultures of micro-organisms: large-scale preparation by continous-flow size selection. Journal of General Microbiology 88, $153-158$.

Mitchison, J. M. (1971). The control of division. In The Biology of the Cell Cycle, pp. 20I-249. Cambridge University Press.

PoOLE, R. K. (1975). Respiration in synchronous cultures of a normal and a respiratory-deficient mutant strain of Escherichia coli. Proceedings of the Third European Workshop on the Cell Cycle, Edinburgh, September, I975, p. II.

Poole, R. K. (1977). Fluctuations in buoyant density during the cell cycle of Escherichia coli KI 2 : significance for the preparation of synchronous cultures by age selection. Journal of General Microbiology 98, 177186.

PoOLE, R. K. \& HADDOCK, B. A. (1975). Dibromothymoquinone: an inhibitor of aerobic electron transport at the level of ubiquinone in Escherichia coli. FEBS Letters 52, $13-16$.

Poole, R. K. \& LLOYD, D. (1974). Changes in respiratory activities during the cell cycle of the fission yeast Schizosaccharomyces pombe $972 \mathrm{~h}^{-}$growing in the presence of glycerol. Biochemical Journal $\mathbf{1 4 4}, \mathrm{I}_{4} \mathrm{I}-148$.

POOLE, R. K., LLOYD, D. \& KEMP, R. B. (1973). Respiratory oscillations and heat evolution in synchronouslydividing cultures of the fission yeast Schizosaccharomyces pombe $972 \mathrm{~h}^{-}$. Journal of General Microbiology 77, 209-220.

SCHAIRER, H. U. \& HADDOCK, B. A. (1972). $\beta$-Galactoside accumulation in a $\mathrm{Mg}^{2+}, \mathrm{Ca}^{2+}$-activated ATPase deficient mutant of E. coli. Biochemical and Biophysical Research Communications 48, 544-55I.

Scopes, A. W. \& Williamson, D. H. (I964). The growth and oxygen uptake of synchronously-dividing cultures of Saccharomyces cerevisiae. Experimental Cell Research 35, 36I-37I.

SMITHER, R. (1975). Use of a Coulter counter to detect discrete changes in cell numbers and volume during growth of Escherichia coli. Journal of Applied Bacteriology 39, I37-165.

StePhenson, M. (1949). Growth and Nutrition. In Bacterial Metabolism, 3rd edn, pp. 159-178. London: Longmans Green \& Co.

ZARITSKY, A. \& PRITCHARD, R. H. (1973). Changes in cell size and shape associated with changes in the replication time of the chromosome of Escherichia coli. Journal of Bacteriology 114, 824-837. 